\title{
A Workaround of EHR - A logistics/Reporting System Development
}

\author{
Sonya H.Y. Hsu \\ School of Computing and Informatics \\ University of Louisiana Lafayette \\ SonyaHsu@louisiana.edu
}

\author{
Steven Dick \\ Picard Center \\ University of Louisiana Lafayette \\ Dick@louisiana.edu
}

\begin{abstract}
This project presents a use case - The Lab (TL) services multiple hospitals, medical centers, and physicians' offices in the southern area of the United States. Applying systematic methods of business process management, the project manager and development team clarify requirements, analyze the processes, develop logistics and create a reporting system for TL. The system must be designed to retrieve data with limited time and costs for an inundated EHR system. In this project, the authors try to define the logistic requirements of $T L$ and the needs an electronic form for an information management system. For example, automate processes and eliminate waste. Besides the EHR software, the goal of this project is to improve web-based logistics and reporting system while maintaining HIPAA compliant controls. The project achieves the goals, but the workaround system is still cumbersome yet workable.
\end{abstract}

\section{Introduction}

Since 2011, the incentive program "meaningful use" (MU) of IT encouraged the adoption of clinical practices to use better IT such as providing quality patient care, service providers, and patients communication, shortening service gaps. The MU program is meant for those clinical practices offered to Medicare and Medicaid patients. When the MU program rolled into its third stage 2015 to 2017, the adoption rate should have increased exponentially. In reality, MU Stage 2 produced disappointing results while small independent practices (less than five physicians) tend to be slow in adoption $[5,10]$.

EHR is systematized collection of patient and population health information in a digital format. These records can be shared across different health care settings. As of July 2016, 175 health IT vendors supplied certified EHR products to 4,474 (4567 in 2016) hospitals participating in the Centers for
Medicare and Medicaid Services (CMS) EHR Incentive Programs. CMS is a federal agency within the United States Department of Health and Human Services that administers the Medicare program and works in partnership with state governments to administer Medicaid. Also, CMS's responsibilities include the administrative simplification standards from the Health Insurance Portability and Accountability Act of 1996 (HIPAA), and other quality standards, such as long-term care facilities (nursing homes), clinical laboratory, and oversight of HealthCare.gov [28]. The key features of the current EHR system are embodied in the Software as a Service (SaaS) platform including on premise, charting, labs and imaging, patient portal, e-prescription, workflow automation, appointment scheduling and mobile accessibility [2]. In other words, SaaS is so-called "on-demand" software that clients may pick and choose what is needed in the line of business. In recent years, "revenue management" seems to have taken the essential role in the EHR as companies like Cerner, MEDITECH, Epic, Evident, McKesson, and MEDHOST Systems take nearly $92 \%$ market share of primary certified EHRs for participating hospitals [22]. Within the CMS HER Incentive program, 337, 432 (490,575 in 2016) health care participating providers, Epic Systems, Allscripts, eClinicalWorks, Athenahealth, and NextGen Healthcare, comprise over $60 \%$ market share of primary certified EHRs for participating professionals [23]. Epic Systems offer to $30 \%$ of all those participating providers in the Incentive Program.

For most small medical practices, the set-up costs of adopting new or updating the Electronic Health Records (EHR) systems are high. So high, those practices rather take the possible penalty for noncompliance to the MU program. On average, one small medical center with five physicians (50 to 70 patients from each physician per day) and their EHR system costs around US\$23,000 each month [12]. Whenever the government requires some new standards and changes, it adds on to the existing system with 
additional costs on the module(s) chosen. The investment in an EHR system may not be justified based on a return on performance incentive for the independent or small clinical practices. Noncompliance can cause a practice to lose up to $10 \%$ of Medicare Part B reimbursements [19].

Hoffman [10] stated the complexity and cost of developing, implementing, and managing the technology meant that American health-care providers - most of whom work in small practices with fewer than five physicians - found little reason to adopt it [10].

Considering change-over implementation, costs, training and end-users' involvement, plenty of the medical centers plan to continue to use old systems as long as possible. Older systems, such as Cerner, have been slowly updated if at all. Lots of hospitals still use the inundated version of Cerner or other legacy EHRs. For example, TL is affiliated with a major hospital, and several satellite medical centers and physician's offices continue to use Cerner after more than ten years due to change costs and time. The front-line providers (i.e., physicians, nurses, specialists, lab, imaging and other technical, clerical and operations staff) must understand EHR protocol and hopefully, utilize it to enhance their tasks at hand [11]. All the changes, including professional development about "updated" of EHR system, changing workflow disruptions and interoperability problems [30], require resources.

Currently, medical service providers who cannot afford upgrade costs must wait a little longer while managing their practices. TL is such a case. In this project, the authors try to define the logistic requirements of TL and the needs an electronic form for an information management system. For example, automate processes and eliminate waste. Besides the EHR software, the goal of this project is to improve web-based logistics and reporting system while maintaining HIPAA compliant controls.

\section{Literature review}

The value of EHR can be divided into productivity and value produced. The maximum productivity that EHR can achieve within an interval of time is a function of effectiveness, efficiency, and flexibility [27] where the relationship between EHR effectiveness and efficiency is mediated by flexibility. EHR value would be based on the accumulation of digitized patient data but moderated by EHR input, cost and labor hours to obtain these data [27]. The EHR productivity is highly relevant to both process management and meaningful use of EHR. Productivity is not a linear function over time. As the useful life of an EHR system diminishes, the costs and labor to maintain the system increase exponentially. Multiple modules of EHR system replacement become too expensive, the hospitals and affiliated medical centers must work around the system and adapt to get the job done. Webster and Copenhaver [27] used the framework of the closed-looped control system to conceptualize business process management protocols that increase the effectiveness and efficiency of an EHR system. From the Table 1, the service-oriented architecture (SOA) applies to this case: pick and choose, then configure and customize. SOA complements with SaaS, loosely couples with different modules that are required in business processes and operations.

A high-quality interface is necessary, Swanson and Lind [26] specifically indicated "Different practices and healthcare facilities have different processes, so a system may test well in one context and poorly in another (p. 57)." With eight challenges in implementing and usability testing, they support "summative tests" that are comparable and meaningful across different context and systems. In one case [7], Healthcare.gov failed because the scope of the project is far more complex than expected and then rushed to completion without enough of an implementation planning and testing. Simply put, summative testing would prevent the disastrous outcome from the Canadian based consulting firm responsible for Healthcare.gov [7]. Further, the interfaces of EHR must be clinically relevant and realistic so that practitioners can understand its use and relationship to their work. The modernize enterprise EHR is more of an interdisciplinary workflow system than a standalone data recording tool [11]. Starting 2011, Usability Evaluation Protocol (UEP) by National Institute of Standards and Technology (NIST) sought to standardize summative usability testing for all EHR products with the goal of increasing patient safety and reducing errors [20, 26].

Standardized technology provides some stability to user's acceptance while maintaining certain quality control. On the other hand, the standardization can become a cumbersome compliance issue. A dynamic interface between users and information systems is necessary but the methodology to achieve a userfriendly information system can be a difficult goal to achieve and measure. Khare et al. [14] proposed a user interface to provide flexible Electronic Health Record (fEHR) system, which allows clinicians to build new templates/forms for data collection over an existing EHR. The system automatically translates the forms to underlying databases while shielding the user from need-to-know technical details. The fEHR corrects the 
rigidity of current EHR system and provides a personalized interface for each medical practices. This interface transforms the structured database into customized forms. The fEHR system is form-based in two ways: collecting the clinicians' requirements to extend of the database; transforming the information from the filled form in the reflection of clinicians' needs to the database.

\subsection{Barriers in Implementing HER}

Holden and Davidson [11] questioned the efficacy of MU program and some possible unintended consequences. They also stressed the possible detrimental effects on small practices (i.e., primary care and rural practices) that "continue to face high barriers" to the EHR adoption and assimilation. The barriers may differ from each organization size, its type, practice locations, and resources (e.g., financial, staff, and IT support). Such small practices may be most affected by the lack of qualified health IT support services is one of the targets that was identified in the article by Blumenthal [2].

Interoperability can be an enormous barrier while implementing and assimilating the "new" EHR system. Most EHR systems are NOT communicative with each other [12]. Sometimes, even within the same system between organizations or practices in different adoption period will have interoperability problems (e.g., the legacy vs. updated). HIPPA restrictions, which minimize data sharing, may create interoperability issues - especially with patient data. Blackman [1] has proposed a plan to achieve interoperability through a technical fix within different EHR systems. One known medical group which houses more than 20 physicians, 200 employees, and various practices, uses GE Centricity for the medical practices, e-ClinicalWorks for revenue management, and a third party system to manage patient check-in [12]. This medical group needs its own IT staff for channeling communication and local troubleshooting while an outsourced IT company does the needed "heavy lifting" to achieve interoperability. Interoperable systems are only useful when they function as expected. In reviewing 86 journal publications regarding electronic medical records adoption, Ludwick and Doucette [18] indicated "socio-technical" factors that influence health information systems deployment greatly. The sociotechnical factors entice the interactions between technical and social features that further stress the importance of "people" factor.

Data interoperability between health systems is critical to providing efficient patient care to improve the accuracy of diagnoses, reduction in duplicated tests, minimize readmission, and prevent medication errors [8]. One of the major causes of electronic health information interoperability issues is the heterogeneity of clinical data sources that operate on the foundation of data standard models restricting the exchange of data to external domains [9]. The functional specification of the design will capture the flow of data from the EHR systems (data input) to the clinical repository (transformed data output). The process design specification will demonstrate the flow of data from the EHRs to the integration engine to the mapping and translation model [1].

A couple of studies focused on the logistics process with EHR using Web Ontology LanguageDescription Logic (OWL-DL) [13, 16]. Jing et al. [13] intended to establish a standardized prototype to manage customized information from the information exchanges using manual and automatic methods. Their project developed the integration of customized information based on existing patient record and individual patient characteristics. From Landry and Philippe's [16] project, all information from EHR, and the customized information from the knowledge base were displayed automatically via the EHR interface. The EHR interface was a combination of front-end interfaces, back-end tables associated with EHR. Qureshi and Noteboom [25] focused on offering physicians the best available evidence about the most effective treatment for their patients. A combination of data from tests and biomedical databases on a condition is crucial for any medical treatment [25]. Providing the lab results in a timely fashion is one instrumental link in evidence-based treatment. TL intends to keep up with time-pressured tasks with their clients. In this case, if TL can manage its lab in further details, such as scheduling the pathologists, nurses and lab technicians, that can streamline their workflows in processing lab requests.

This research lies in the design of a systematic approach to the motivation of the process manager who allows radical performance improvement via two IT prototypes and redesigned processes to increase competitiveness. This research focuses on the development of a logistic/reporting system around the existing EHR system in a pathology lab. A systematic method is used to collect requirements, workflow and business processes.

With these research focuses, this manuscript is organized in the followings: use case, collected requirements, workflow redesign, implementation and a conclusion. 


\section{Use case}

TL is a sub-specialty anatomic pathology lab in a southern United States city (population 250,000). TL specializes in Cytopathology, Dermatopathology, Forensic Pathology, G.I. \& Liver Pathology, Hematopathology, Molecular Genetic Pathology, and Oncological Pathology servicing hospitals with more than 300 beds and 4,500 employees. In addition, TL is contracted to regional medical centers, physicians' offices, Forensic Center and coroner's office. Each year, TL has 20,000 to 22,000 cases of tissues processing and 40,000 cases of Cytology for OBGYN. Several hospitals in the network require TL adopting EHR system to improve communication. While being compliant to the contracted services, TL can neither track specimens nor generate specimen reports over any time frame (daily, weekly or monthly). The greatest concern is missing specimen incidents leading toward TL's inability to keep a 24 to 48 -hour turnaround promise.

With the current system, compiling timely information is inefficient or not possible. If there is a need for a report, a request sent from the IT department of the contracted hospitals can take from seven to fourteen days. However, if the report generated in the TL, it would have taken the head nurse 45 minutes to query and another 2 hours to generate the report of a one-week period, but it may not come out as exactly needed by the manager to make a decision.

"Tissue Examination Request" form is crucial and must be matched with specimens, testing, and diagnosis every step of the way. Any "missing" specimens or forms triggers a process to go back to "find missing specimen" (in Figure 1) to double check all pathology lab forms and trace back to the ordering doctor's office.

Compiling information is labor intensive and slow. If there is a need for a missing specimen report, the flow from the IT department to the contracted hospitals takes from seven to fourteen days. However, if the report generated via HER in TL, it would take the head nurse 45 minutes to query and another 2 hours to produce the report of a one-week period but may not come out exactly as needed by the manager to make a decision.

The basic workflow (see Figure 1) starts with physicians' orders from surgical/hospitals/medical centers. Ordering facilities send samples to receiving labs where technicians run requested tests on the samples. Pathologists make diagnoses on the tests results. Then, lab nurses type in diagnoses to the EHR database and reports are sent back to the ordering physicians' offices.

\subsection{Data collections}

The research teams had a kickoff meeting and four site-visits to TL: (1) the first site visit concentrated on the requirements and expected acceptance criterions for this TL projects, two parts were identified: one was the interface for data inputs and outputs, the other was the data reporting function. (2) the first to second site visits included observations of the process of receiving specimens, analyzing and reporting the results to the EHR system. The focuses were the role of EHR, the speed, and function of the data processing in the EHR systems. (3) The third visit was for fine tuning and collecting ideas for improvement in the application design. (4) The last visit was to deliver the final product and the specific step-by-step redesigned processes. The research team also visited the third party technology firm which handled TL's IT needs to coordinate the networking and data dump issues. Besides observations and site visits, the research teams also conducted in-depth interviews with the office manager, medical consultant, head nurse, lab technician and pathologies to collect the requirements of the redesigned system. Each visit and interview took place from 60 minutes to 90 minutes during September to November 2016. Between meetings and visits, there were some short meetings for verifications and clarifications.

Insert Figure1. As-Is logistics process of testing in TL

\section{Collected Requirements}

TL's basic requirements are to view/update records and make/print reports as necessary. The electronic submission of "Tissue Examination Request" form is to allow the medical center nurses to input patient and specimen information into the form, which when submitted, is stored in the database. More detailed requirements such as:

1. Unique patient identifiers to establish the database correlated to multiple incoming specimens from their corresponding patient(s) and ordering physicians.

2. More details/fields to describe patient, specimen, ordering physicians, ordering facility, along with TL's results/prognoses.

3. Check boxes tracking the specimen in each process/step, serve as matching points to correct information, and preventing misplaced specimens through the process.

4. Reporting function to gain knowledge of the workflow quantity. 
In Table 2, there are distinct differences in cycle time after the redesign of the logistic system of TL the cycle time reduced from 690 minutes to 258 minutes. The principal reductions of cycle time include removal of form generation, waiting for the courier, and finding missing specimens.

Insert Table 2. As-is and can-be cycle time

Regarding reporting system, the requirements are to display all of the patient information, the doctor's initial diagnosis as well as the results from the lab test whenever and whichever range of time needed. As indicated, the EHR system takes a long away from the nurse who needs to do additional biological testing instead of waiting 2 to 3 hours to generate a report. In conjunction of file pathology labs electronically, the reporting system is an add-on for the decision-making process regarding scheduling of lab attendants, runners, and pathologists. follows:

The objectives of the reporting systems are as

1. Take control of the data autonomy;

2. Determine referring physicians' orders, look at patterns, extract diagnoses;

3. Try to correlate between what doctor's orders, tissue samples, and testing procedures;

4. Manipulate existing HER data:

a. Reducing wait time in running reports by lab nurses;

b. Reducing requests via IT department generating the report from centralized database;

c. Automate extract, load and transformation (ETL) to be more efficient.

\section{Workflow redesign}

Figure 2 shows the functional activity flowchart after the implementation of the paperless tissue examination request system. The doctor's nurse can now enter all information electronically reducing repeated transcription. In doing so, TL nurses have all information needed ahead of time and to view what and how many specimens to expect for the day. This preview can manage schedules, reduce time and waste. When the specimens arrive, TL nurse can now print labels that match the specimens, instead of having to check each request on paper. Once everything is correct, TL nurses can process the request, and edit the existing information in the database. This information will then transfer to the EHR system.

The major changes between as-is and can-be processes are the removal of form generations, waiting for the courier, and finding missing specimens. These changes reduce the cycle time by 432 minutes. This workaround mainly redesigns by an add-in web-based method for building the database at the point of requesting lab service(s). From digital footprint, it is more efficient to match specimens and more effective to locate missing specimens. Therefore, the office manager can know the quantity of incoming lab services and schedule accordingly.

Insert Figure 2. Can-Be logistics process of testing in TL

An interoperable system allows the information to flow from input to the ordering and even the scheduling of employees. As the TL nurse inputs the diagnosis results in the EHR system it can be viewed by the ordering physicians in addition to the paper form of pathologist's diagnosis. However, the EHR system security limits the necessary exchange information, diagnosis, and patients' data but nothing further. Retrieving the information about how many specimens have been processed and which physician orders tests more often than the others would be cumbersome. Without up-to-date information, the manager has a difficult task to forecast the upcoming strategic planning.

To help TL make more strategic decisions on the specimen reports, a dashboard design is created over the existing Health Level 7 (HL7) files. HL7 refers a model for Open Systems Interconnection (OSI) - specifically for moving clinical and administrative information [21]. While this standard has largely been superseded, the current documents can be accessed in a less than efficient manner. The needed workaround is first to extract data from the HL7 files and into a database. This database is then, connected to a front end of the EHR, anyone with access right would be able to manipulate the data in creating reports and assessments as desired. The result would be a better user interface that works effectively in multiple departments.

\section{Implementations}

The implementation plan for the logistics system. The nurse in the operating room of the medical center will enter the patient and specimen information into the form designed with PHP. The form will be connected to the TL EHR database. Once the data is submitted, it is stored in the EHR database. The TL employees, who are the end users, can access and view the data that is submitted by requesting medical center nurse(s). TL employees or superusers will also be able to update or modify the receiving/processing/traveling 
specimen information. They will be able to generate a report whenever they need to view the incoming specimen samples. A new web interface will be used to access specimen and patient information. All information will be stored on the TL' private server (instead of the centralized database in the contracted hospital) with HL7 transformation to MySQL data dump. The website design is simple and easy for employees to use, update, and view information and generate reports.

\subsection{Logistic system}

For the implementation, a database and web server will be hosted using TL local machine (or a third-party cloud services), which will help maintain HIPAA-compliant regulations. TL uses an Apache server and MySQL database. The local testing environment consisted of a XAMPP client running PHP 5.5. XAMPP is easy to install, a reliable transition to the live version runs on PHP environment.

Programming languages \& tools used in this logistics system are as follows:

1. HTML, CSS, Javascript - Web languages;

2. Bootstrap - Web framework for site layout and styling;

3. jQuery - Javascript library used by Bootstrap;

4. PHP - Scripting language for backend processing;

5. MySQL - Database;

6. PDO - PHP extension for connecting to databases;

7. Apache - Locally hosted development server

Table 2 illustrates the different cycle times between the 'as-is' (615 minutes) and the 'canbe' (275 minutes) processes. The 'can-be' processes (bold type) will save TL a total of 340 minutes or almost 6 hours for each transitional specimen with the redesign. The new design reduces a significant amount of wait time, especially in the form storage, courier wait and specimen location.

Insert Table 2. Cycle time in as-is \& can-be

\subsection{Reporting system}

The report generation begins with the requesting physician order which the patient information was entered and followed by pathologist's diagnosis, physician's decision into EHR system. The EHR data is a digital format accessible to corresponding doctors and labs. For an easy reporting system, the HL7 will be converted into a Comma Separated Value (CSV) file and stored into TL's separate directory.

Programming languages \& tools used in this reporting system are as follows.

1. Apache: Primary server software... used to host all web requests from front to backend;

2. MySQL: storage of data;

3. Phpmyadmin: GUI used to manage backend database and authentication;

4. Java: Used for database parser;

5. HTML: Used to manage visual flow of webpage;

6. PHP: The functions and variable assignments were written within the PHP files, and the majority of variables were formed from the MySQLi statements within the PHP code;

7. MySQLi: Statements used to pull and populate requested data from the SQL database were written within MySQL statements;

8. CSS: Language used to change the visual output of our webpages;

9. FileZilla: open source file management system used to interact with our PC's and the TL file servers;

10. SonicWALL VPN Client: used to establish our VPN connection across to the TL network.

During developing reporting system from the HL7, the conversion from HL7 to CSV is cumbersome. The page break of each patient in HL7 is the issue. Parsing the patient data took three arrays to automate the separation of the patient data. The cycle time to generate the report is in real time rather than waiting the IT department to pull the query in the EHR system. The saving wait time is about 7 to 14 days depending on the complexity and urgency of the queries. The detailed patient data displaced in the front end interfaces which are the result of searching and compilation from the search results queried through the SQL database.

The database includes all patients, attending/ordering physicians, and pathologist information that were listed in the EHR system in HL7 format. These four tables in the Entity-Relationship diagram (ERD; in Figure 3) are linked through the report table which can be generated. The physicians and pathologist all have automated incrementing primary keys. Each time a new physician or pathologist is entered, a column for the newly entered name will be added to the table and given an ID to be referenced in the report table. On the contrast, each patient has a specific account number, so we chose that element as the patient's primary key. As for the Report table attributes, the Accession Number on the top of 
each HL7 file will serve as the primary key. Once the data is extracted from the HL7 files, queries can be made to generate reports on the spot rather waiting for up to 14 days from the centralized IT department from the hospital. In this ER diagram (Figure 3), the primary keys, attending physicians order (APO), pathologist account (PATHOD), patient account (Account), and ordering physician order (OPD) serve as foreign keys in the report (AccessNO).

\section{Conclusion}

This manuscript focuses on the workaround EHR system to improve daily operations in TL. Without changes the legacy system would run out of its useful life. To control upgrade costs, a workaround of the inundated EHR system becomes the choice to streamline business processes in TL.

The proposed changes offer a new interface that dramatically increases ease of use for all constituents while protecting patient privacy. Moreover, a new interface will stand between the disparate systems providing interoperability. Finally, the new system will allow TL to track individual specimens and overall trends allowing a data driven approach to lab management. There is reason to believe that the changes will help TL, its staff, ordering doctors, and patients as well.

The logistics and reporting system is a good attempt to work around the existing inundated EHR system. As in any business process management, there are always rooms for improvements. A retrospecitive analysis, this project focuses on the lean development to reduce waste of time, costs and labor. The impact of which improves the financial results indirectly realized through non-financial operations [24]. That being said, business processes should be monitored, benchmarked and continuously improved. As the project to moves forward, few recommendations should be followed.

1. The current logistics system is designed only to work with one of the surgical centers. With an expectation, the prototype would be tested and expanded one by one until all of TL's affiliations being set up with the standardized system.

2. The back end system needs to be fine-tuned and fully connected. This will ensure that the data is correctly parsed and maintain the data integrity while generating the report. It requires testing before full implementation.

3. The project should not end until the workaround systems can connect, extract, transform, and load (ETL) from EHR system to a private server.
This is the best solution which may linger for most of small to medium medical practices until better solutions come along. If there is continuous improvement, then, the business process should be monitored and benchmarked with some standard key performance index [15].

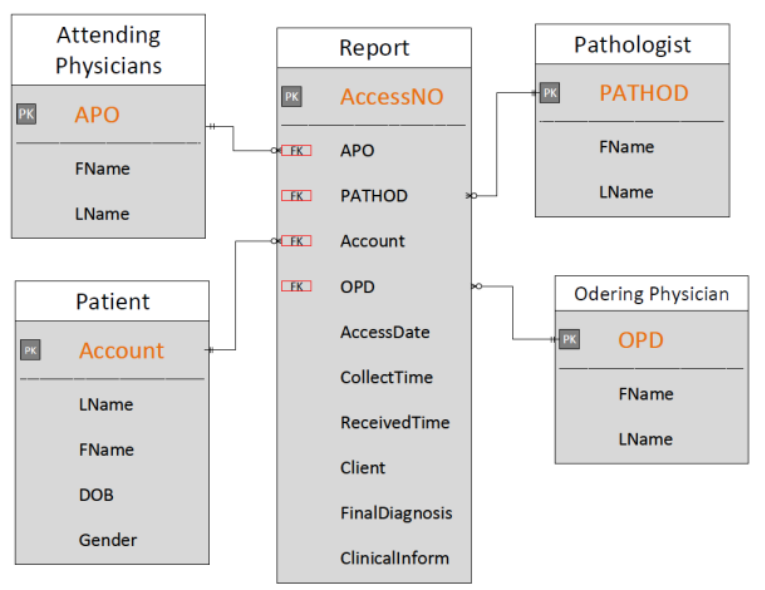

Figure 3: ERD of reporting system

\section{References}

[1] Blackman, S. M. (2017). "Towards a Conceptual Framework for Persistent Use: A Technical Plan to Achieve Semantic Interoperability within Electronic Health Record systems", Proceedings of the 50th Hawaii International Conference on System Sciences, pp. 4653-62.

[2] Blumenthal, D. (2010). "Launching HITECH", New England Journal of Medicine, 362:5, 382-385.

[3] Businss-Software.com. "Top 10 Electronic Medical Record software report: Comparison of the leading EMR softwarevendors."'http://landing.business-software.com/top10-electronic-health-record-software-vendors-v4.php?track $=2122 \&$ traffic $=$ GoogleSearch\&keyword $=$ ehr $\% 20$ vendors $\&$ gclid=Cj0KEQjw1 v66BRCV-6rh6s-Biu8BEiQAelpui2RLs Rcin2tc4TD7GG6aqe4aYqe53hW_hT8uSr_ebKYaAnjc8P 8HAQ assessed April 1, 2017.

[4] Chen, R., Kumar, V., Fitch, N., Jagadish, J., Zhang, L., Dunn, W., Chau, D.H. (2015). "explICU: A web-based visualization and predictive modeling toolkit for mortality in intensive care patients". 37th Annual International Conference of the IEEE Engineering in Medicine and Biology Society (EMBC), pp. 6830 - 6833, DOI: 10.1109/EMBC.2015.7319962.

[5] Davidson, E. and Heslinga, D. (2007). "Bridging the IT adoption gap for small physician practices: An action research study on electronic health records", Information Systems Management, 12:1, 15-28.

[6] Deng, X., Lin, W.H., Tai, Shyong, E., Hao, K.Y., Salloway, M.K., Seng, T.C. (2016). "From descriptive to diagnostic analytics for assessing data quality: An application to temporal data elements in electronic health 
records", IEEE-EMBS International Conference on Biomedical and Health Informatics (BHI), pp. 236 - 239, DOI: 10.1109/BHI.2016.7455878.

[7] Evans, Brad (2014). The Obamacare Website. Ivey Publishing.

[8] Furukawa, M. F., Patel, V., Charles, D., Swain, M., \& Mostashari, F. (2013). "Hospital electronic health information exchange grew substantially in 2008-12", Health affairs (Project Hope), 32(8), 1346-1354.

[9] Gabriel, M. H., Jones, E. B., Samy, L., \& King, J. (2014). "Progress and challenges: Implementation and use of health information technology among critical-access hospitals", Health Affairs, 33(7), 1262-1270.

[10] Hoffman, L. (2009). "Implementing Electronic Medical Records", Communications of the ACM, 52(11): 18-20.

[11] Holden, K., Davidson, E. (2017). "Sorting out HER adoption and assimilation in the meaningful use incentive program", in Hawaii Proceedings of the 50th Hawaii International Conference on System Sciences, 3421-30.

[12] Hsu, S., Thakur, R., Dick, S. (in progress). The myths of EMR implementations.

[13] Jing, X., Kay, S., Marley, T., Hardike, N.R. (2014). "Integration of an OWL-DL knowledge base with an EHR prototype and providing customized information", Journal of Medical Systems, 38(9): 1-14.

[14] Khare, R., An, Y., Song. I.Y., Hu, X. (2010). "Can clinicians create high-quality databases? A Study on a Flexible Electronic Health Record (fEHR) System”, IHI'10, November 11-12, 2010, Arlington, VA.

[15] Koetter, F., Kochanowski, M. (2015). "A model-driven approach for event-based business process monitoring", Information System E-Business Management, 13(1):5-36.

[16] Landry, S., Philippe, R. (2004). "How Logistics Can Service Healthcare", An International Journal of Supply Chain Forum, 5 (2): 24-30.

[17] Leeper D. (2012). “Obamacare's Fatal Flaws: Complexity and Central Planning”, Tea Party Tributes, July 6, $2012 \quad$ http://www.teapartytribune.com /2012/07/06/obamacares-fatal-flaws-complexity-andcentral-planning/ August 30 ${ }^{\text {th }}, 2017$.

[18] Ludwick, D.A. and Doucette, J. (2009). “Adopting Electronic Medical Records In Primary Care: Lessons Learned From Health Information Systems Implementation Experience In Seven Countries", International Journal of Medical Informatics, 78(1): 22-31.

[19] Massachusetts eHeath Institute. http://mehi.masstech.org/support/ehealth-

education/ehealth-impact-drivers/macra-mips assessed August $30^{\text {th }}, 2017$

[20] NIST. (2017). Health IT Usability. https://www.nist.gov/programs-projects/health-it-usability. Assessed June $12^{\text {th }}, 2017$.
[21] NIST. (2017). HIT Transactional testing. https://www.nist.gov/itl/ssd/transactional-testing Assessed June $12^{\text {th }}, 2017$.

[22] Office of the National Coordinator for Health Information Technology. (2016). 'Electronic Health Record Vendors Reported by Hospitals Participating in the CMS EHR Incentive Programs,' Health IT Quick-Stat \#29. dashboard.healthit.gov/quickstats/pages/FIG-Vendors-ofEHRs-to-Participating-Hospitals.php. Accessed May 30 2017.

[23] Office of the National Coordinator for Health Information Technology. (2016). 'Electronic Health Record Vendors Reported by Health Care Professionals Participating in the CMS EHR Incentive Programs and ONC Regional Extension Centers Program,' Health IT Quick-Stat \#30. dashboard.healthit.gov/quickstats/pages/FIG-Vendorsof-EHRs-to-Participating-Professionals.php. Accessed May 302017.

[24] Peronja, I. (2015). "Performance Effects of the Business Process Change In Large Enterprises: The Case Of Croatia", Management: Journal of contemporary management issues, 20 (1): 1-22.

[25] Qureshi, S., Noteboom, C. (2017). "Knowledge Activation for Patient Centered Care: Bridging the Health Technology Divide", Proceedings of the 50th Hawaii International Conference on System Sciences, pp. 931-9.

[26] Swanson, A., Lind, S. (2011). "Usability Testing EHRs: Example from the Front Lines”, ACM Special Topic, pp. 5458.

[27] Webster, C., Copenhaver, M. (2010). "Process-Aware EHR BPM Systems: Two Prototypes and a Conceptual Framework", Studies in Health Technology and Informatics, 160 (Pt 1): 106-110.

[28] Wikipedia, Center of Medicare and Medicaid, https://en.wikipedia.org/wiki/Centers for Medicare and Medicaid_Services last accessed August 30 ${ }^{\text {th }}, 2017$.

[29] Wikipedia, Software as a Service, https://en.wikipedia.org/wiki/Software_as_a_service last accessed August 30 ${ }^{\text {th }}, 2017$.

[30] Wright, A., Henkin, S., Feblowitz, J., McCoy, A. B., Bates, D. W., \& Sittig, D. F. (2013). "Early Results of the Meaningful Use Program for Electronic Health Records", New England Journal of Medicine, 368(8), 779-780. 
Table 1. Ranking of top 10 EMR/EHR vendors

\begin{tabular}{|c|c|c|c|c|c|c|c|c|c|c|c|c|}
\hline Modules & descriptions & vendor & $\begin{array}{c}\text { Medi } \\
\text { tech }\end{array}$ & $\begin{array}{c}\text { GE } \\
\text { EMR }\end{array}$ & $\begin{array}{c}\text { Green } \\
\text { way }\end{array}$ & $\begin{array}{c}\text { Care } \\
360 \\
\end{array}$ & $\begin{array}{c}\text { McKe } \\
\text { sson }\end{array}$ & $\begin{array}{c}\text { All } \\
\text { scripts }\end{array}$ & $\begin{array}{c}\text { Inter } \\
\text { Systems }\end{array}$ & $\begin{array}{l}\text { eClinic } \\
\text { Works }\end{array}$ & Cerner & Epic \\
\hline \multirow[t]{5}{*}{ Physician tools } & \multicolumn{2}{|l|}{ E-prescribing } & 85 & 85 & 100 & 85 & 85 & 85 & 85 & 85 & 100 & 85 \\
\hline & \multicolumn{2}{|c|}{ Medication tracking } & 85 & 85 & 70 & 70 & 70 & 85 & 85 & 85 & 0 & 70 \\
\hline & \multicolumn{2}{|l|}{ CPOE } & 85 & 85 & 70 & 70 & 70 & 85 & 70 & 85 & 0 & 70 \\
\hline & \multicolumn{2}{|c|}{ ICD-10 Support } & 100 & 100 & 100 & 100 & 100 & 100 & 100 & 100 & 100 & 100 \\
\hline & \multicolumn{2}{|l|}{ ONC-ATCB } & 100 & 100 & 100 & 100 & 100 & 100 & 100 & 100 & 100 & 100 \\
\hline \multicolumn{3}{|c|}{ Average } & 91.00 & 91.00 & 88.00 & 85.00 & 85.00 & 91.00 & 88.00 & 91.00 & 60.00 & 85.00 \\
\hline \multirow[t]{4}{*}{ Patient centric } & \multicolumn{2}{|c|}{ Patient Portal built in } & 100 & 100 & 100 & 100 & 100 & 100 & 100 & 100 & 100 & 100 \\
\hline & \multicolumn{2}{|c|}{ Patient Portal $3^{\text {rd }}$ Party } & 85 & 100 & 85 & 85 & 85 & 85 & 85 & 85 & 100 & 70 \\
\hline & \multicolumn{2}{|c|}{ Appointment Scheduling } & 85 & 100 & 85 & 85 & 85 & 85 & 85 & 85 & 85 & 70 \\
\hline & \multicolumn{2}{|c|}{ Automatic Reminder } & 100 & 100 & 100 & 100 & 100 & 100 & 100 & 100 & 100 & 100 \\
\hline \multicolumn{3}{|c|}{ Average } & 92.50 & 100.00 & 92.50 & 92.50 & 92.50 & 92.50 & 92.50 & 92.50 & 96.25 & 85.00 \\
\hline \multirow[t]{4}{*}{ Reporting } & \multicolumn{2}{|c|}{ Lab Orders \& Results } & 85 & 85 & 100 & 85 & 85 & 70 & 85 & 70 & 85 & 70 \\
\hline & \multicolumn{2}{|l|}{ Immunization } & 85 & 85 & 85 & 70 & 85 & 70 & 70 & 0 & 70 & 70 \\
\hline & \multicolumn{2}{|c|}{ Practice Management } & 100 & 85 & 85 & 100 & 85 & 85 & 50 & 85 & 85 & 85 \\
\hline & \multicolumn{2}{|l|}{ Custom } & 85 & 100 & 85 & 100 & 85 & 100 & 85 & 70 & 85 & 85 \\
\hline \multicolumn{3}{|c|}{ Average } & 88.75 & $\mathbf{8 8 . 7 5}$ & 88.75 & 88.75 & 85 & 81.25 & 72.5 & 56.25 & 81.25 & 77.5 \\
\hline \multirow{3}{*}{$\begin{array}{l}\text { Providers } \\
\text { Interoperability }\end{array}$} & \multicolumn{2}{|c|}{ Source Direct Messaging } & 85 & 85 & 85 & 100 & 85 & 100 & 85 & 85 & 85 & 85 \\
\hline & \multicolumn{2}{|l|}{ E/M Coding } & 85 & 70 & 85 & 85 & 85 & 70 & 50 & 70 & 70 & 70 \\
\hline & \multicolumn{2}{|c|}{ Insurance Verification } & 85 & 85 & 85 & 85 & 100 & 70 & 85 & 85 & 100 & 0 \\
\hline \multicolumn{3}{|c|}{ Average } & 85.00 & 80.00 & 85.00 & 90.00 & 90.00 & 80.00 & 73.33 & 80.00 & 85.00 & 51.67 \\
\hline \multirow{2}{*}{$\begin{array}{l}\text { Auxiliary } \\
\text { features }\end{array}$} & \multicolumn{2}{|c|}{ Doc Uploading/Storage } & 85 & 70 & 85 & 85 & 100 & 85 & 100 & 100 & 70 & 85 \\
\hline & \multicolumn{2}{|l|}{ Mobile Apps } & 100 & 100 & 100 & 100 & 100 & 100 & 100 & 100 & 100 & 100 \\
\hline $\begin{array}{l}\text { Architectural } \\
\text { Feature }\end{array}$ & \multicolumn{2}{|c|}{ Hosted Installations } & 100 & 100 & 100 & 100 & 100 & 100 & 100 & 100 & 100 & 100 \\
\hline \multicolumn{3}{|c|}{ Overall Average } & 90.53 & 90.53 & 89.74 & 89.74 & 89.74 & 88.16 & 85.26 & 83.68 & 80.79 & 79.74 \\
\hline
\end{tabular}

Table 2. As-Is and Can-Be cycle time

\begin{tabular}{|c|c|c|c|c|c|c|c|c|c|c|c|c|c|}
\hline \multirow{2}{*}{\begin{tabular}{|l|} 
As-is \\
Fill out request \\
\end{tabular}} & \multirow{2}{*}{\begin{tabular}{|l|} 
Can-Be \\
Fill out request \\
\end{tabular}} & \multicolumn{2}{|c|}{\begin{tabular}{|l|}
$\begin{array}{l}\text { Processing } \\
\text { time }\end{array}$ \\
\end{tabular}} & \multicolumn{2}{|c|}{ Wait Time } & \multicolumn{2}{|c|}{$\begin{array}{l}\text { Move } \\
\text { Time }\end{array}$} & \multicolumn{2}{|c|}{$\begin{array}{l}\text { Inspection } \\
\text { time }\end{array}$} & \multicolumn{2}{|c|}{$\begin{array}{l}\text { Updating } \\
\text { Time }\end{array}$} & \multicolumn{2}{|c|}{ Total Time } \\
\hline & & 10 & 3 & & & & & & & & & 10 & 3 \\
\hline Request Pickup & Send requests & & & 10 & 1 & & & & & & & 10 & 1 \\
\hline Store/send forms & & & & 120 & & & & & & & & 120 & \\
\hline Store specimen & Store specimen & & & 60 & 60 & & & & & & & 60 & 60 \\
\hline Wait for Courier & Wait for Courier & & & 240 & 120 & & & & & & & 240 & 120 \\
\hline \begin{tabular}{|l|} 
Delivery \\
\end{tabular} & Delivery & & & & & 30 & 30 & & & & & 30 & 30 \\
\hline Specimen arrival & Specimen arrival & 5 & 5 & & & & & & & & & 5 & 5 \\
\hline & Print report & & 2 & & & & & & & & & & 2 \\
\hline $\begin{array}{l}\text { Matching with } \\
\text { specimen }\end{array}$ & $\begin{array}{l}\text { Matching with } \\
\text { specimen }\end{array}$ & 5 & 5 & & & & & 30 & 5 & & & 35 & 10 \\
\hline $\begin{array}{l}\text { Find missing } \\
\text { specimen }\end{array}$ & $\begin{array}{l}\text { Find missing } \\
\text { specimen }\end{array}$ & & & 120 & 20 & & & & & & & 120 & 20 \\
\hline $\begin{array}{l}\text { Process exam } \\
\text { request }\end{array}$ & $\begin{array}{l}\text { Process exam } \\
\text { request }\end{array}$ & 30 & 5 & & & & & & & 30 & 2 & 60 & 7 \\
\hline & & & & & & & & & & & & 690 & 258 \\
\hline
\end{tabular}

*Can-Be processes in bold type. 


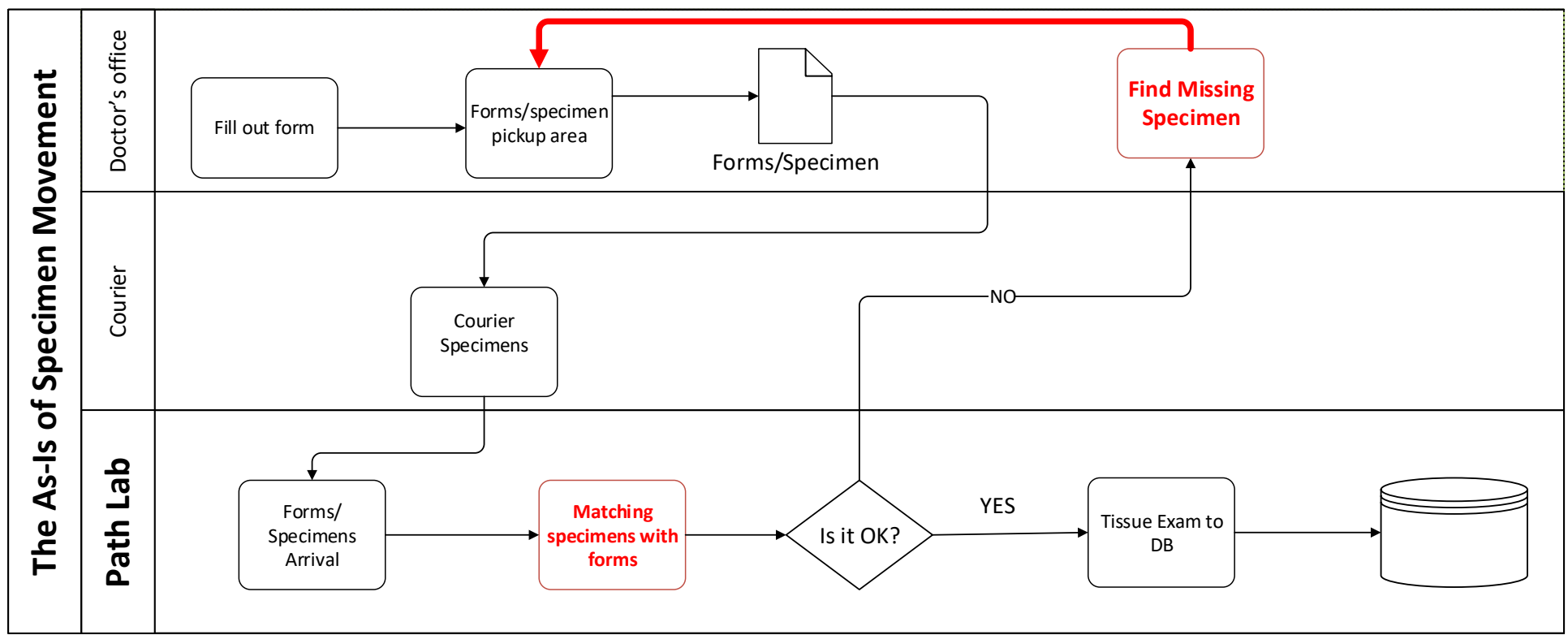

Figure 1. As-Is Logistics Process of Testing in TL

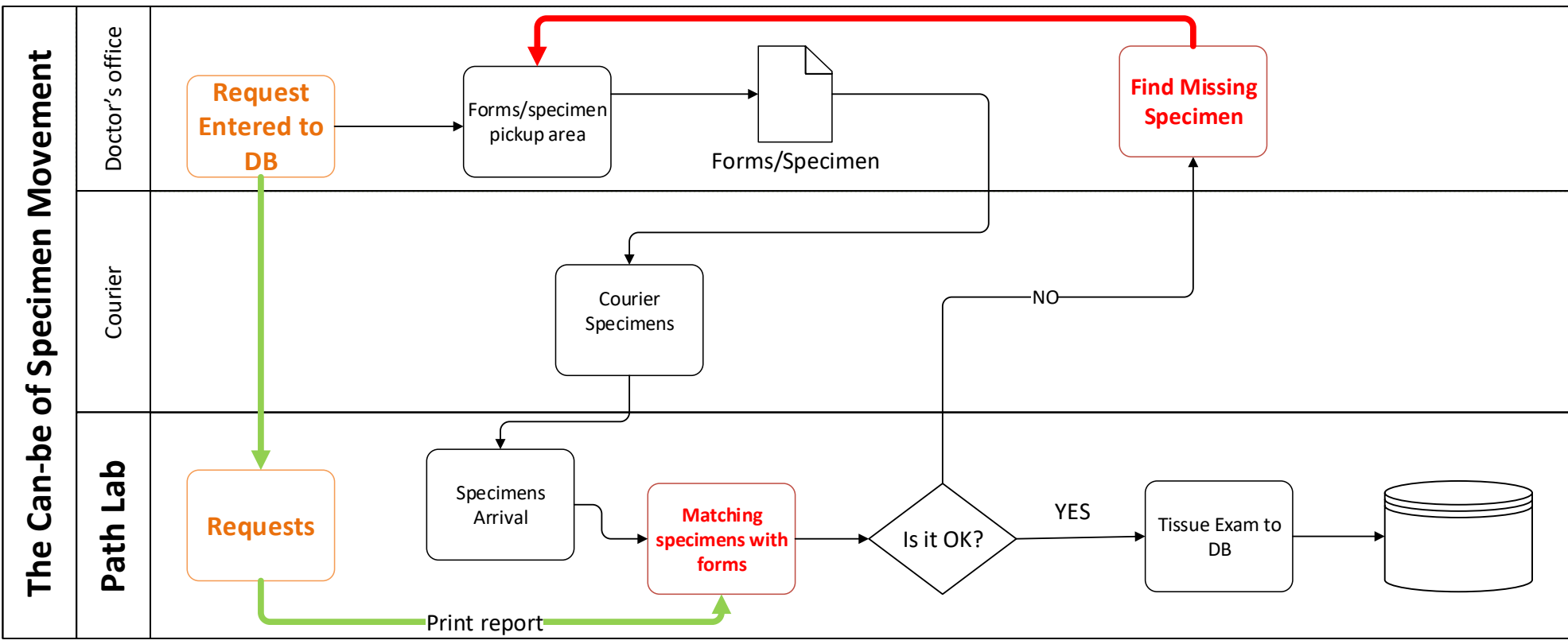

Figure 2. Can-Be Logistics Process of Testing in TL 